\title{
Die Akutbehandlung von Sportverletzungen am Spielfeldrand
}

\author{
Raymond Best, Mario Bucher
}

\section{Zusammenfassung}

Eine besondere Herausforderung der sportmedizinischen Tätigkeit stellt die Betreuung von sportlichen Großveranstaltungen, Spitzensportbetreuung im Individual- und Mannschaftssport und nicht zuletzt die Spielfeldrandbetreuung einer Teamsportsart dar. Das Erkennen, Diagnostizieren, Einschätzen, Behandeln und Managen von akuten Sportverletzungen, die während eines Wettkampfs oder Spieles auftreten, stellt aufgrund des meist bestehenden Zeitdrucks sowie der besonderen äußeren Umstände sicherlich eine Sonderform der ärztlichen Arbeit dar. Neben medizinischer Expertise bedarf es zur adäquaten Behandlung derartiger Sportverletzungen daher einer guten Vorbereitung, eines organisatorischen Geschicks, eines feinfühligen Umgangs mit allen direkt und indirekt beteiligten Personen und Branchen und nicht zuletzt, trotz aller Einflussfaktoren, einer ärztlichen Neutralität, um im Verletzungsfall dem Wohl und dem Schutz des Athleten immer die höchste Priorität einzuräumen. Der vorliegende Artikel soll daher die verschiedenen Blickwinkel und Probleme der Behandlung von Sportverletzungen am Spielfeldrand im Sinne eines Erfahrungsberichts aus der Sicht des Teamarztes beleuchten. Aufgrund seiner großen Verbreitung und Beliebtheit in Mitteleuropa gilt dem Fußballsport dabei ein besonderes Augenmerk.

\section{Treatment of Sport Injuries at the Sidelines}

Being a "doctor on the sidelines" of a sportive mass participation event or a premier league team event as well as being asked to be an athlete's or a team's sport physician certainly is a special challenge of a sports physician's activity. Due to the pressure of time as well as the particular external circumstances, recognizing, diagnosing, evaluating, treating and managing competition-associated acute sports injuries might be a special form of medical acting. To deal adequately with such injuries, besides a medical expertise it requires a good preparation, organizational skills, a delicate handling of all direct and indirect involved parties - and not least a medical neutrality despite all external influencing factors, to give the wellbeing of the athlete the highest of all priorities. The present article describes the different perspectives and problems whilst treating such acute injuries from a team physician's viewpoint. Hereby, due to its widespread popularity in central Europe, the article especially focuses on soccer team sports.

\section{Einleitung}

Bei der Akutbehandlung von Sportverletzungen in der Sportorthopädie/Sporttraumatologie stellt die Betreuung von Sportveranstaltungen - und hier im Besonderen eine direkte Spielfeldrandbetreuung - für sportmedizinisch tätige Ärzte und Physiotherapeuten aus medizinischer und ethischer Sicht eine besondere Herausforderung dar [1]. Dies ist vor allen Dingen der Tatsache geschuldet, dass schnelle medizinische Ad-hocEntscheidungen im Falle von Verletzungen oder medizinischen Zwischenfällen während eines Einzel- oder Mannschaftswettkampfs am Spielfeldrand unter ganz anderen Voraussetzungen getroffen werden müssen als bei der ärzt-

OP-JOURNAL 2016; 32: 10-14

(C) Georg Thieme Verlag KG Stuttgart · New York DOI http://dx.doi.org/10.1055/s-0042-106761 lichen Tätigkeit im Praxis- oder Klinikalltag [2]. Neben den naturgemäß begrenzten diagnostischen Mitteln vor Ort können äußere Faktoren wie Zeitdruck, Erwartungshaltung seitens des Trainers und des Athleten, Interessenkonflikte und nicht zuletzt die mediale und örtliche Aufmerksamkeit einer Vielzahl von Zuschauern die Entscheidung eines spielbetreuenden Teamarztes erheblich beeinflussen [3].

Zum Beispiel ist die Durchführung einer validen Untersuchung der Kniegelenksstabilität vor Tausenden von Fans, laufender Kamera und somit Hunderttausenden von Zuschauern eine gänzlich andere Erfahrung als im vertrauten und ruhigen Umfeld der eigenen Untersuchungsräume in Klinik und Praxis [2] (Abb. 1). Erschwerend folgt der Untersuchung des Spielers auf dem Feld unmittelbar eine ausgesprochen hohe $\mathrm{Er}$ wartungshaltung aller Beteiligten (Ath- let, Trainer, Verein, Medien) $[4,5]$ in Bezug auf eine verlässliche und zweifelsfreie Diagnose und Ausfallzeit. Unklare oder zweifelhafte Befunde sind nur schwer $\mathrm{zu}$ vermitteln und generieren schnell Unsicherheit [6], was den behandelnden Arzt weiter „unter Druck“ setzt.

Nichtsdestoweniger sollen und müssen, trotz der genannten extrinsischen deutlich druckerhöhenden Faktoren, die medizinischen und ethischen Standards bei der Befund- und Diagnoseerhebung gleich angesetzt werden wie im klinischen Alltag [7]. Die alles entscheidende Frage der Schwere einer aufgetretenen Verletzung und der sich daraus ergebenden Entscheidung der weiteren Spielfähigkeit des Athleten erfordert daher integeres, neutrales und objektives medizinisches Handeln mit einem sicherlich höheren Maß an ärztlicher Erfahrung als es auf den ersten Blick erscheinen mag. 


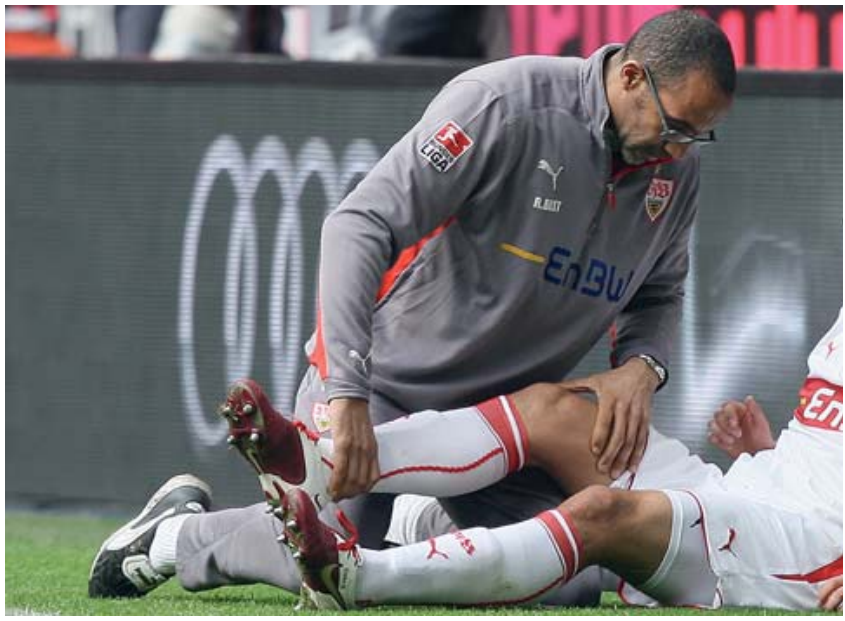

Ziel des vorliegenden Übersichtsartikels ist es daher, die verschiedenen Blickwinkel und Probleme der Behandlung von Sportverletzungen am Spielfeldrand im Sinne eines Erfahrungsberichts aus teamärztlicher Sicht zu beleuchten. Neben den rein medizinisch-inhaltlichen Aspekten der einzelnen Fachrichtungen soll ein besonderes Augenmerk auf die Begleitumstände gelegt werden, welche die ärztliche Tätigkeit eines Teamarztes am Spielfeldrand entscheidend prägen. Bedingt durch die sportartspezifische Popularität sowie eigene Erfahrungen konzentriert sich der Artikel dabei im Wesentlichen auf den Volkssport Nr. 1 in Mitteleuropa, den Fußballsport.

\section{Epidemiologie von akuten Sportverletzungen}

Generell muss die Inzidenz von akuten Sportverletzungen im Allgemeinen und während eines Wettkampfs gesondert voneinander betrachtet werden. Beispielsweise konnte gezeigt werden, dass sich im Fußballsport das Verletzungsrisiko in der Wettkampfsituation im Vergleich zum normalen Mannschaftstraining in etwa verfünffacht [8]. Dies ist u.a. der höheren Anspannung sowie der höheren Risikobereitschaft unter Wettkampfbedingungen geschuldet. Statistische Erhebungen der Fußballweltmeisterschaften 2002 und 2006 ergaben zudem eine weitere Verdopplung des Verletzungsrisikos während eines großen internationalen Turniers im Vergleich zum nationalen Ligaalltag [8]. Während es sich bei den meisten spielassoziierten Akutverletzungen dabei um kleinere Bagatelltraumen handelt, führt nur etwa jede 5.-10. Verletzung im Sinne einer „major time loss injury“ zu einer Spielausfallzeit eines Athleten von mehr als 4 Wochen [8].
Abb. 1 Untersuchung eines Kniegelenks am Spielfeldrand im Rahmen eines Fußballbundesligaspiels.

Naturgemäß variieren Auftreten und Anzahl von wettkampfassoziierten Sportverletzungen in Abhängigkeit zur jeweils ausgeübten Sportart, wobei Sprunggelenks-, Knie- und Kopfverletzungen neben den Muskelverletzungen zu den häufigsten Sportverletzungen zählen [9]. Mannschaftssportarten mit Körperkontakt gelten als sog. High-Impact-Sportarten zu den verletzungsträchtigsten überhaupt [7]. Statistiken konnten das erhöhte Verletzungsrisiko bei den Sportarten Handball, Basketball und vor allem Fußball im Vergleich zu Individualsportarten wie Laufen oder Tennis im europäischen Raum belegen [3].

Eine prospektive Kohortenstudie bez. der Verletzungsanzahl bzw. des Verletzungsrisikos aller 14 Mannschaftssportarten der olympischen Sommerspiele 2004 positionierte das Verletzungsrisiko im Fußball bspw. an 2. bzw. 3. Stelle [10]. Bemerkenswerterweise zeigt sich in den letzten 2 Jahrzehnten eine deutliche Zunahme von Nichtkontaktverletzungen gegenüber den Kontaktverletzungen, während sich die Verteilung der Verletzungsart bzw. Verletzungslokalisation kaum geändert hat $[3,11,12]$. Ein Teamarzt scheint somit besonders bei Wettkämpfen im Mannschaftssport mit überwiegend kleineren Verletzungen besonders gefordert.

Interessanterweise prägen jedoch nicht die in hoher Anzahl auftretenden kleineren Verletzungen, als eher die selteneren, schwereren Verletzungen aufgrund der gesteigerten medialen Aufmerksamkeit die Wahrnehmung der ärztlichen Tätigkeit eines Mannschaftsarztes am Spielfeldrand. Während 35\% aller Verletzungen im Fußball die Muskulatur betreffen [13], erlitten nach einer Datenbankrecherche der Fußballbundesliga im Zeitraum von 6 Spielzeiten (2009-
2014) „lediglich“ 46 Spieler eine Ruptur des vorderen Kreuzbands, entsprechend durchschnittlich 7,6 Kreuzbandrupturen in der gesamten 1. Fußballbundesliga pro Saison $[14,15]$. In nahezu allen Fällen erfuhr der Teamarzt hierbei eine deutlich übergeordnete mediale Aufmerksamkeit vom Moment der ersten Verdachtsäußerung. Nichtsdestoweniger besteht die Hauptaufgabe eines Mannschaftsarztes während seiner Spielfeldrandbetreuung vergleichsweise selten in der akuten Diagnoseerhebung eines vorderen Kreuzbandrisses, als vielmehr in der Beurteilung von Prellungen, muskulären Verletzungen, Sprunggelenkstraumen oder Ähnlichem.

Zusammenfassend soll hiermit aufgezeigt werden, dass die größte Verantwortung eines Mannschaftsarztes an der Seitenlinie vor allen Dingen darin besteht, schwerwiegende bzw. höhergradige Verletzungen mit einer möglichen nachhaltigen Schädigung des Sportlers schnell und sicher zu erkennen und diese aus der Vielzahl kleinerer Bagatelltraumen während eines Spielbetriebs „herauszufischen“. Gerade weil das beobachtende Umfeld im Moment der ärztlichen Spielerbegutachtung aus Gewohnheit, eigener Erfahrung („im Zweifel sitz es aus“ [7]) sowie gewissem Eigeninteresse immer von kleineren Verletzungen ausgeht, erfordert eine objektive Verletzungsselektion ein gewisses Fingerspitzengefühl.

\section{Vorbereitende Maßnahmen}

Auch wenn es auf den ersten Blick nebensächlich erscheint: Einer der Hauptaspekte der Akutbehandlung von Sportverletzungen seitens des spielfeldrandbetreuenden Mannschaftarztes ist ein Vorbereiten auf den Akutfall an sich [7]. Anders als im Praxis- und Klinikumfeld bestehen beim Auftreten einer Sportverletzung in oft fremdem Umfeld keine bekannten örtlichen Pfade, keine schnell verfügbaren diagnostischen Ressourcen oder gar wortlose Absprachen im Falle therapeutischer Interventionen.

Im Zweifelsfall muss sich der Sportarzt jedes Wochenende auf neue, teilweise unbekannte Gegebenheiten einstellen, derer er sich im Vorfeld bewusst werden muss. So sollte er sich bspw. mit der Infrastruktur fremder Stadien sowie der umgebenden Krankenhauslandschaft vertraut machen. Er sollte geklärt haben, auf welchem Wege er einen schwerer verletzten Athleten einem 
Krankenhaustransport zuführt bzw. auf welche Möglichkeiten er vor Ort zurückgreifen kann (Behandlungsräume, Tragen, Stretche, Vakuummatratzen etc.). Insbesondere weil der (mediale) Fokus im Fall einer höhergradigen Verletzung auf dem spielfeldrandbetreuenden medizinischen Team liegt, muss dieses hier mit höchster Verantwortung bereits im Vorfeld agieren [7].

Hilfreich ist hierbei grundsätzlich die Kontaktaufnahme zum Teamarzt der entsprechenden Heimmannschaft für einen Informationsaustausch und unterstützende Absprachen. Die eigene Erfahrung hat dabei gezeigt, dass es durchaus sinnvoll ist, sich dabei nicht nur auf die Vorbereitung o.g. Sportverletzungen zu beschränkten, sondern gerade bei Massenveranstaltungen auch medizinisch fachfremde Sondersituationen im Hinterkopf zu behalten (z. B. Herz-KreislaufBeschwerden oder Sturz bei Zuschauern oder dem Betreuerstab), die schnell in den Verantwortungsbereich eines Teamarztes fallen können.

\section{Akutbehandlung während des Spieles}

Grundsätzlich sollte man als Mannschafts- bzw. Teamarzt das Spiel aufmerksam verfolgen, um den Unfallmechanismus vor einer Verletzung, wenn möglich, direkt zu beobachten. So können bereits erste Schlüsse zur Schwere der Verletzung gezogen werden [6].

Im Falle einer spielunterbrechenden Verletzung ist es zunächst der Schiedsrichter, der sich einen ersten Eindruck verschafft und daraufhin bez. der Notwendigkeit des Einsatzes eines Teamarztes auf dem Feld entscheidet. Hierbei wird man beobachten, dass dieser trotz einer „eindrucksvollen“ Verletzung oft besonnen und ruhig die subjektiven Beschwerden des Spielers aufnimmt und vergleichsweise selten die Unterstützung des Mediziners in Anspruch nimmt.

Doch auch wenn höhergradige oder gar katastrophale Verletzungen während des Spielbetriebs ausgesprochen selten sind [16], sollte jede Verletzungssituation aus Sicht des Teamarztes eher übervorsichtig als zu schnell verharmlosend beurteilt werden. Im Zweifelsfalle ist es - rein ärztlich betrachtet - schwerwiegender, eine relevante Verletzung in der Kürze der Zeit zu übersehen und einen Spieler somit durch Weiterführen des Spieles nachhaltig zu schädigen, als einen Befund fälschlicherweise überzu- interpretieren und im Sinne der Gesundheit des Spielers zu viel Vorsicht walten zu lassen.

Grundsätzlich jedoch haben gerade professionelle Sportler ein ausgesprochen gutes Eigenempfinden für physische Störungen, sodass deren Äußerung auf dem Feld in den meisten Fällen ein sehr verlässlicher erster Gradmesser für die Schwere der aufgetretenen Verletzung ist $[6,17,18]$.

Folglich beschränkt sich die erste ärztliche Anamnese daher meist auf die Fragen „was ist passiert, wie schlimm ist es?" und vermittelt in der knapp vorhandenen Zeit auf dem Rasen oft einen sehr genauen ersten Eindruck. Die Entscheidung über die weitere Spielfähigkeit ist dennoch nicht immer einfach, da auch die subjektive Einschätzung des Spielers z.B. von psychologischen Faktoren entscheidend beeinflusst werden kann. Jung et al. [19] beschreiben verschiedene Persönlichkeitsmerkmale bei Athleten, die bspw. zur „Competitive Anxiety“ (Wettkampfangst) oder zu „General Wellbeing“ (alles kein Problem) neigen, und deren persönliche Einschätzung der Situation möglicherweise überoder untertreibt.

Rein objektiv gesehen kann auf dem Spielfeld das Auftreten eines der folgenden, einfach und schnell zu eruierenden Faktoren auf eine mögliche spielfähigkeitslimitierende Verletzung hinweisen $[6,17,18]$ :

- subjektive Einschätzung des Sportlers selber

- Gelenkfehlstellung

- Gelenkschwellungen

- subjektive oder objektive Instabilität

- größere blutende Wunden

- anhaltender oder gar zunehmender Schmerz

- anhaltende subjektive oder objektive asymmetrische Belastungsfähigkeit

- eingeschränkter muskulärer Kraftaufbau

- mangelnde Orientierung oder gar stattgehabter Bewusstseinsverlust

In allen genannten Fällen bedarf es vor einer finalen Entscheidung über eine weitere Spielfähigkeit, so denn diese nicht sofort auf dem Feld geklärt werden kann (bzw. muss), einer eingehenden Untersuchung außerhalb des Spielfelds. Zeitdruck besteht aufgrund der Unterzahl der eigenen Mannschaft auch hier, er muss jedoch nun eine untergeordnete Rolle spielen.
Therapeutisch gesehen interveniert der Mannschaftsarzt zur Erlangung einer Spielfähigkeit nur durch wenige, vom PECH-Schema bekannte Maßnahmen (Eis, Kompression). Die Gesellschaft für Orthopädisch-Traumatologische Sportmedizin (GOTS) stellt zudem mit ihren Ausgaben der Expertenmeetings aus den Jahren 2010, 2012 und 2014 bereits Leitfäden zur Behandlung von Sprunggelenks-, Knie- und Schulterverletzungen auf dem Spielfeld zur Verfügung, die ebenfalls Berücksichtigung bei der „OnField“-Betreuung finden $[6,17,18]$. Ein weiterer Leitfaden zur Behandlung von Muskelverletzungen ist in Vorbereitung.

Nach Entscheidung zum Auswechseln oder Weiterspielen wird der Spieler in letzterem Fall dann vom Schiedsrichter wieder auf das Feld gerufen. Wichtig ist, dabei zu beachten, dass der Spieler Schmerzen haben kann, humpeln darf oder gar nur stehen kann - blutende Wunden müssen jedoch im Sinne einer „Infectious Disease Policy“ [20] vor Betreten des Spielfelds bluttrocken versorgt sein (Bandage oder Naht), blutige Spielkleidung muss gewechselt werden [6] (Abb. 2).

Eine Sonderregelung der Verletzungsbehandlung gilt seit Kurzem bei Kopfverletzungen im Fußballsport. Häufig noch werden diese von Spielern und betreuendem Arzt unterschätzt (Abb.3). Einem Athleten sollte nach einer Kopfverletzung jedoch in jedem Fall erst dann eine Rückkehr aufs Feld zum Weiterspielen gestattet werden, wenn eine mögliche, höhergradige Verletzung mit an Sicherheit grenzender Wahrscheinlichkeit ausgeschlossen werden kann [21]. Da bspw. eine „simple“ Gehirnerschütterung in gewisser Weise erst in Ruhe eine retrospektive Diagnose vorübergehender neurologischer Symptome nach entsprechendem Trauma ist [7], sollte die Freigabe zur Weiterführung eines Spieles nach Kopfverletzung eher zurückhaltend erteilt werden. Beispielhaft wurde dies vor einem Millionenpublikum während der Fußballweltmeisterschaft 2014, als ein Spieler der DFBAuswahl nach versehentlichem Kopfstoß das Spiel zunächst weiterführte - kurz darauf jedoch nicht mehr wusste, wo er war.

Um die Einschätzung und Versorgung der Spieler trotz der erwähnten zeitlichen Drucksituation besser beurteilen zu können, gibt es im Falle von Kopfverletzungen seit Kurzem nun die Möglich- 


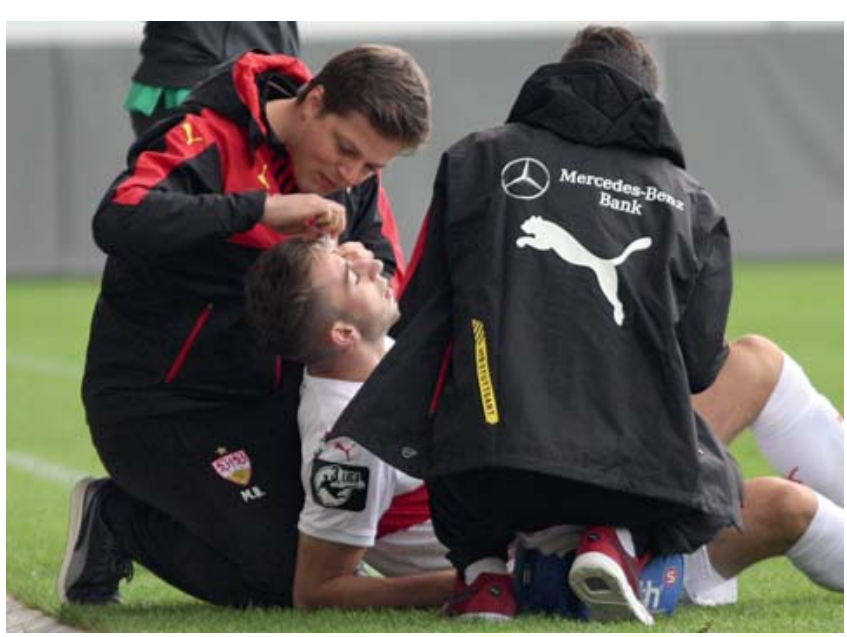

Abb. 2 Spielfeldrandversorgung einer blutenden Wunde im Rahmen eines Ligaspiels.
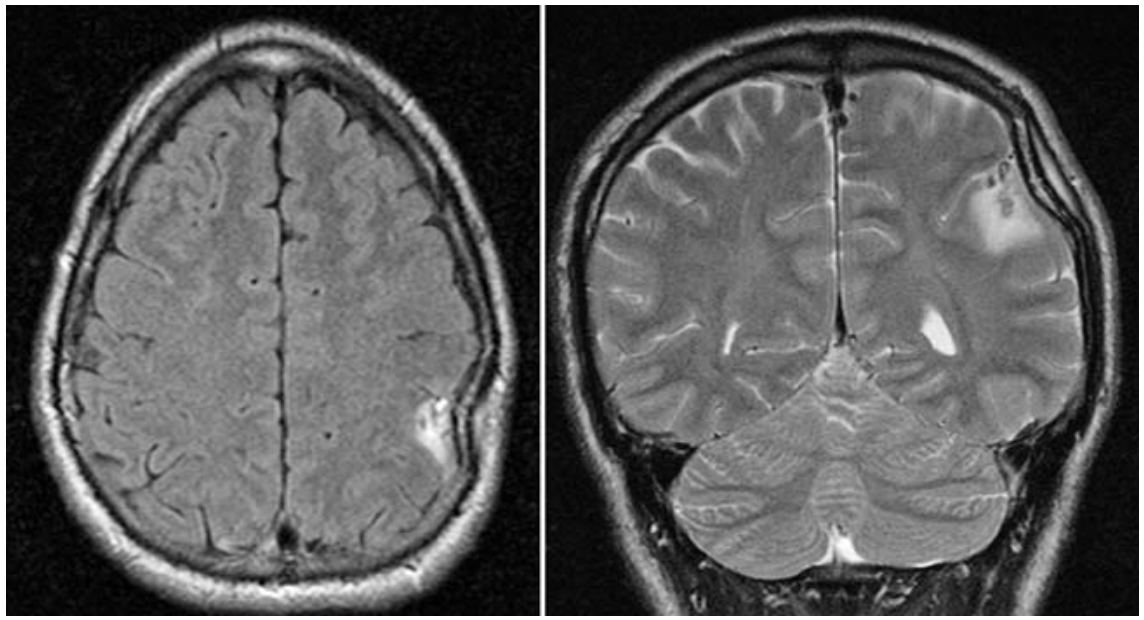

Abb.3 Kleine, protrahiert erkannte Impressionsfraktur parietal. Der Amateurspieler hatte die Verletzung im Rahmen eines Ligaspiels nach Kopfstoß im Rahmen des Kopfballs mit anschließender 10-sekündlicher Bewusstlosigkeit erlitten.

keit einer 3-minütigen Untersuchungsund Behandlungszeit auf dem Feld, in der nur der Arzt allein die Entscheidung über die weitere Spielfähigkeit des Spielers anhand dessen kongnitiver Fähigkeiten trifft [22].

Diese Entscheidung kann bei Verdacht auf Gehirnerschütterung anhand des Sports Concussion Assessement Tools Version 3 (SCAT-3) beurteilt werden [23]. Das Tool wurde in Zusammenarbeit der internationalen Fußball-, Rugby- und Eishockeyverbände sowie dem Internationalen Olympischen Komitee (FIFA, IRB, IIHF und IOC) konzipiert. Inhalt sind Testungen der Orientierung zur Zeit, der Konzentration, der Koordination, des Erinnerungsvermögens und des Gleichgewichts, zusätzlich wird der GlasgowComa-Scale erfasst. Da der SCAT-3-Test in der Durchführung 15-20 Minuten beansprucht, ist er in der Vollversion während eines Spieles natürlich nicht praktikabel. Anwendung kann deshalb nur die modifizierte Version finden, das PocketRecognition-Tool, das mit einer Durchführungsdauer von maximal 1 Minute rasch bessere Klarheit bringt [23].

Zusätzlich empfehlen McCrory et al. [24] eine sofortige Krankenhauseinweisung mit entsprechender Bildgebung bei den in Tab. 1 auftretenden Symptomen. Eine eingehende neuropsychologische Untersuchung ist hingegen nur bei den in Tab. 2 vorliegenden Befunden empfohlen.

Zusammenfassend kann gesagt werden, dass die Hauptaufgabe eines Teamarztes bei der Versorgung von Sportverletzungen auf oder neben dem Spielfeldrand vor allem das schnelle und adäquate Erkennen und Managen einer Verletzung ist [7]. Das unbedingte Vermeiden nachhaltiger Schädigungen eines Spielers ist dabei genauso wichtig wie das Zulassen „normaler“ leistungssportassoziierter schmerzhafter Begleiterscheinungen
Tab. 1 Symptome, die zur sofortigen Einweisung in ein Krankenhaus führen sollten [7].

\begin{tabular}{l} 
- Verlust des Bewusstseins für länger als \\
5 Minuten \\
\hline $\begin{array}{l}\text { - zunehmende Kopfschmerzen, Schwin- } \\
\text { del oder Erbrechen }\end{array}$ \\
\hline - verletzungsassoziierter Krampf \\
\hline - ungleiche Pupillen \\
\hline - veränderte kardiovaskuläre Parameter \\
(z.B. deutlich erhöhter Blutdruck) \\
\hline - neurologische Auffälligkeiten
\end{tabular}

Tab. 2 Symptome, die eine eingehende neuropsychologische Untersuchung zur Folge haben sollten [7,24].

- Verlust des Bewusstseins für länger als 5 Minuten

- posttraumatische Amnesie für mehr als 24 Stunden

- verletzungsassoziierter Krampf

- Symptome einer Gehirnerschütterung für mehr als 2 Wochen

- wiederkehrende Gehirnerschütterungen

[3]. Und auch wenn Zeitdruck, Publikum, Wichtigkeit eines Spielers oder eines Spieles kaum zu ignorierende Einflussgrößen vor und während einer Spielbetreuung darstellen [3], so dürfen aus ärztlicher Sicht in der Entscheidungsfindung nur die aus Klinik- bzw. Praxisalltag gewohnten medizinisch-ethisch und moralisch einwandfreien Grundsätze gelten, um der Verantwortung als Teamarzt zweifelsfrei gerecht zu werden [2].

\section{Schweigepflicht}

Bei der Spielfeldrandbetreuung ergibt sich häufig die Situation, dass gerade bei schwereren Verletzungen Trainer, Verein und nicht zuletzt die Medien vor Ort kurz nach der Untersuchung eine erste Auskunft zu Art und Schwere einer Verletzung erfragen. Wie im normalen Arzt-Patienten-Verhältnis unterliegt man jedoch auch bei der Betreuung von Sportlern der ärztlichen Schweigepflicht. Prinzipiell ist durch den Sportler die Entbindung von der Schweigepflicht notwendig, um den Vereinsverantwortlichen (oder Medien) medizinische Befunde mitteilen zu dürfen [25]. Es bietet sich deshalb an, im Rahmen der Vertragsunterschrift oder des Medizinchecks 
eines Athleten eine Schweigepflichtentbindungserklärung gegenüber den Vereinsverantwortlichen schriftlich zu fixieren. Diese Erklärung kann vom Sportler jederzeit, auch mündlich, widerrufen werden. In diesem Fall darf und muss der Arzt im Verletzungsfall die Vereinsverantwortlichen informieren, dass er vom Sportler nicht oder nur in Teilen von der Schweigepflicht entbunden wurde. Beim Umgang mit den Medien empfiehlt sich aus eigener Erfahrung ein zurückhaltendes Auftreten, es ist ratsam die Kommunikation mit den Medien in enger Absprache mit dem Pressesprecher des Vereins zu führen und somit selbst eher im Hintergrund zu agieren.

\section{Fazit}

Die sportärztliche Betreuung einer Sportveranstaltung oder einer Mannschaft ist eine größere Herausforderung, als es auf den ersten Blick scheinen mag und geht weit über das oft mit einem Schmunzeln zu sehende „Eis- und Handauflegen" hinaus. Bei der Behandlung von einer akuten Verletzung geht es dabei weniger um eine sofortige „Wundertherapie“ zur Wiederherstellung der Sportfähigkeit eines Athleten, als vielmehr um das schnelle und sichere Erkennen, Einschätzen und Selektieren der Art und Schwere der Verletzung vor den Augen und Erwartungen einer gelegentlich nahezu grenzenlosen Anzahl von Beteiligten und Teilnehmenden. Neben der rein medizinischen Qualifikation bedarf es einer guten Vorbereitung und eines organisatorischen Geschicks - und nicht zuletzt, trotz aller äußerer Einflüsse, einer ärztlichen Neutralität, um im Verletzungsfall dem Wohl und dem Schutz des Athleten immer die höchste Priorität einzuräumen.

\section{Literatur}

1 Stovitz S, Satin D. Professionalism and the ethics oft the sideline physician. Curr Sports Med Rep 2006; 5: 120-124

${ }^{2}$ Krajca-Radcliffe J, Cummings NM. Sideline ethical dilemmas. J Bone Joint Surg Am 2014; 96: e132 [Epub ahead of print]

${ }^{3}$ Best R, Bauer G, Niess A et al. [Return to play decisions in professional soccer - a decision algorithm from a team physician's viewpoint]. Orthop Unfall 2011; 49: 582-587

4 Best TM, Brolinson G. Return to play - the sideline dilemma. Clin J Sports Med 2005; 15: 403-404

${ }^{5}$ Creigthon DW, Shrier I, Shultz R et al. Return to play: a decision based model. Clin J Sports Med 2010; 20: 379-385

${ }^{6}$ Pieper HG, Gokeler A, Krifter RM et al. Spielfeldbetreuung und Behandlung der akuten Instabilität. GOTS-Expertenmeeting Schulterinstabilität 2014; 71-77

7 Verrall GM, Brukner PD, Seward HG. Doctor on the sidelines. Med J Aust 2006; 184: 244-248

${ }^{8}$ Dvorak J, Junge A, Grimm K et al. Medical report from the 2006 FIFA World Cup Germany. Br J Sports Med 2007; 41: 578-581

${ }^{9}$ Henke T, Luig G, Schulz D. Sportunfälle im Vereinssport in Deutschland. Bundesgesundheitsbl Gesundheitsforsch Gesundheitssch 2014; 57: 628-637

10 Junge A, Langevoort G, Pipe A et al. Injuries in team sport tournaments during the 2004 Olympic Games. Br J Sports Med 2006; 40: 193-201

11 Dupont G, Nedelec M, McCall A et al. Effect of 2 soccer matches in a week on physical performance and injury rate. Am J Sports Med 2010; 38: 1749-1751

12 Inklaar $H$. Soccer injuries. I: incidence and severity. Sports Med 1994; 18: 55-73

${ }^{13}$ Ekstrand J. Epidemiologie von Muskelverletzungen im Fußball. In: Müller-Wohlfahrt HW, Hrsg. Muskelverletzungen im Sport. Stuttgart, New York: Thieme; 2014: 150-158

${ }^{14} \mathrm{Im}$ Internet: https://fussballverletzungen. wordpress.com/tag/kreuzbandriss; Stand: 12.04.2016

15 Im Internet: http://www.transfermarkt.de/1bundesliga/startseite/wettbewerb/L1; Stand: 12.04.2016

${ }^{16}$ Australian Government Department of Health and Ageing. Sport safety in Australia: an update: July 2003. Im Internet: http//www. health.gov.au/internet/wcms/publishing.nsf./ content/phd-pub-injury-sportssafety-cnt. htm; Stand: Januar 2006
17 Miltner O, Glaser C, Greitemann B et al. Spielfeldbetreuung. GOTS-Expertenmeeting Sprunggelenk 2012; 17-22

18 Schmitt H, Freiwald J, Engelhardt $M$ et al. Spielfeldbetreuung. GOTS-Expertenmeeting Vorderes Kreuzband 2010; 27-32

19 Junge A, Dvorak J, Roesch D et al. Psychological and sport-specific characteristics of football players. Am J Sports Med 2000; 28 (5 Suppl.): S822-S828

${ }^{20}$ Sports Medicine Australia. Policy: infectious diseases. Im Internet: http://www.sma.org. au/pdfdocuments/infDisease.pdf; Stand: Januar 2006

${ }^{21}$ McCrory P. Concussion - a sports doctor's dilemma: management and return to play issues. N Z J Sports Med 2000; 28: 92-96

22 McCrory P, Johnston K, Meeuwisse W et al. Summary and agreement statement of the 2nd International Conference on Concussion in Sport, Prague 2004. Clin J Sports Med 2004; 15: 48-55

${ }^{23}$ McCrory P, Meeuwisse WH, Aubry M et al. Consensus statement on concussion in sport: th 4th International Conference on Concussion in Sport held in Zurich, November 2012. Br J Sports Med 2013; 47: 250-258

${ }^{24}$ McCrory P, Makdissi M, Davis G et al. Value of neuropsychological testing after head injuries in football. Br J Sports Med 2005; 39 (Suppl. 1): S58-S63

25 Striegel H. Ärztliche Schweigepflicht - Bedeutung in der Leistungssportbetreuung. medical sports network 2015; 5: 8-10

\section{Dr. med. Raymond Best}

Leitender Arzt

Department Sportorthopädie/

Sporttraumatologie Untere Extremität Facharzt für Chirurgie, Orthopädie und Unfallchirurgie, Notfallmedizin, Sportmedizin

Sportklinik Stuttgart

Taubenheimstraße 8

70372 Stuttgart

best.raymond@sportklinik-stuttgart.de 